\title{
Molecular Diagnosis of COVID-19: An Update and Review
}

\author{
Ketan Priyadarshi ${ }^{1}$ Vijaya Lakshmi Nag ${ }^{1} \quad$ Sarika P. Kombade ${ }^{1} \quad$ Ravi Sekhar Gadepalli ${ }^{1}$ \\ Sanjeev Misra ${ }^{2}$ Kuldeep Singh ${ }^{3}$
}

1Department of Microbiology, All India Institute of Medical

Sciences, Jodhpur, Rajasthan, India

${ }^{2}$ Department of Surgical Oncology, All India Institute of Medical

Sciences, Jodhpur, Rajasthan, India

${ }^{3}$ Department of Paediatrics, All India Institute of Medical Sciences, Jodhpur, Rajasthan, India

Ann Natl Acad Med Sci (India):2020;3:126-137
Address for correspondence Vijaya Lakshmi Nag, MD, Department of Microbiology, All India Institute of Medical Sciences, Jodhpur 342005, Rajasthan, India (e-mail: vijayalakshmi005@gmail. com).

\begin{abstract}
SARS-CoV-2 belongs to genus Betacoronavirus subgenus Sarbecovirus of the family Coronaviridae, which originated as a global public health problem. The disease caused is termed as Coronavirus Disease (COVID-19). The virus spread in more than 213 countries and territories all over the world and disease was declared as a pandemic. SARS-CoV-2 is an enveloped positive-sense single- stranded ribonucleic acid (ssRNA) virus. Severe infection and high-mortality are seen in patients with comorbid conditions like diabetes, hypertension, cancer, old age, malnutrition, children, and pregnancy. In India, strategy for testing of COVID-19 has been framed and revised over the course of time by the Indian Council of Medical Research (ICMR), Department of Health Research (DHR), Ministry of Health and Family Welfare (MOHFW), Government of India. ICMR has created a network of viral research and diagnostic laboratories (VRDLs) all over the country, and COVID-19 testing has also been undertaken by ICMR. AIIMS Jodhpur is a regional level VRDL, mentoring 38 government and private laboratories for molecular diagnosis of COVID-19 in the states of Rajasthan and Gujarat. Currently, real-time reverse transcription polymerase chain reaction (rRT-PCR) assay is used all over the country for diagnosis of COVID-19. It uses the TaqMan fluorogenic probe-based chemistry and 5'- nuclease activity of Taq DNA polymerase. It targets specific genes like the RdRp gene, HKU-Orf1 ab gene, E gene, and N gene. Apart from rRT-PCR, other isothermal nucleic acid test (NAT)-like transcription-mediated amplification (TMA), loop-mediated isothermal amplification (LAMP), etc. are emerging diagnostic tools to detect COVID-19.

Keywords

- COVID-19

- SARS-CoV-2

- RdRp and HKU-Orf1ab

- molecular detection

- RTPCR

- NAAT

- diagnostic assays Cepheid Xpert Xpress SARS- CoV-2 test and Truenat betaCoV are point-of-care molecular assays which gives results in less than 1 hour, and helps to provide rapid and accurate results. Automated molecular assays like Cobas SARS-CoV-2 and Multiplex NAAT BioFire Respiratory Panel 2.1 (RP2.1) are newer techniques to curb the disease. Sherlock CRISPR SARS-CoV-2 kit is a highly specific and sensitive assay developed to diagnose COVID19. rRT-PCR has been combined with techniques like bead hybridization, digital droplet $\mathrm{PCR}$, microarray, etc. for improving the correct diagnosis. Recent molecular assays are the future pillars for containment of COVID-19 outbreaks all over the world.
\end{abstract}

\section{Introduction}

Coronaviruses (CoVs) belong to the family Coronaviridae, isolated from various species of birds, snakes, bats, and other mammals. They include four genera-Alphacoronavirus, Betacoronavirus, Gammacoronavirus, and Deltacoronavirus. The endemic CoVs causing the human infection are HCoV-229E and HCoV-NL63 in genus Alphacoronavirus; 
and $\mathrm{HCoV}-\mathrm{OC} 43$ \& HCoV-HKU1 in the A-lineage (subgenus Embecovirus) of genus Betacoronavirus. ${ }^{1,2}$ In 2002, a Betacoronavirus in lineage B (subgenus Sarbecovirus), originating in bats, spread from civets to humans in the Guangdong province of southern China, caused severe respiratory disease, which was given the name Severe Acute Respiratory Syndrome-related CoV (SARS-CoV). ${ }^{3}$ In 2012, a Betacoronavirus in lineage C (subgenus Merbecovirus) spread from camels to humans in Saudi Arabia, causing a similar clinical syndrome as SARS, which was given the name Middle East Respiratory Syndrome-related CoV(MERS-CoV). ${ }^{4}$

On December 31, 2019, a cluster of cases of pneumonia of unknown causes were reported in Wuhan city of China. The novel Betacoronavirus was identified and named as SARS-CoV-2 by the International Committee of Taxonomy of Viruses (ICTV). As per the classification of infectious diseases, the World Health Organization (WHO) named the disease Coronavirus Infectious Disease 2019 (COVID-19). On January 30,2020 , WHO declared it a public health emergency of international concern. Subsequently, COVID-19 has spread over the world across 213 countries and territories. The most affected nations are the USA, Russia, Spain, Brazil, UK, Italy, France, Germany, Turkey, Iran, and India. On March 11, 2020, WHO declared COVID-19 as a global pandemic. ${ }^{5}$

SARS-CoV-2 is an enveloped, positive-sense, singlestranded ribonucleic acid (ssRNA) virus with a diameter of 50 to $200 \mathrm{~nm}$ which comprises four structural proteins, i.e., spike protein (SP), envelope protein (EP), membrane protein (MP), and nucleocapsid protein (NP). It has a crownlike appearance, which is a characteristic of CoVs. The genome encodes 27 proteins including an RNA-dependent RNA polymerase (RdRp). ${ }^{2}$ SARS-CoV-2 has approximately $79 \%$ genome homology with the SARS-CoV and approximately $88 \%$ sequence identity with the bat $\mathrm{CoV}$, but only approximately $50 \%$ homology with the MERS-CoV, and is taxonomically related to the subgenus Sarbecovirus. ${ }^{1,2}$ Genome changes resulting from recombination, gene exchange, gene insertion, or deletion are frequent among CoVs. ${ }^{6}$

Infection is acquired either by inhalation of respiratory droplets or through contact with surfaces contaminated by them. After an incubation period of 2 to 5 days, the patient develops varying degrees of symptoms, ranging from fever, headache, fatigue and myalgia to sore throat, cough and shortness of breath. All ages and sex are susceptible. Severe infection and high-mortality are seen in patients with comorbid conditions like diabetes, hypertension, cancer, lung disease, cardiovascular disease, immunosuppressive drugs, old age and children ${ }^{7}$ leading to complications like acute lung injury, acute respiratory distress syndrome (ARDS), shock, and acute kidney injury. ${ }^{8,9}$

\section{COVID-19 Testing Strategy in India}

In India, a strategy for "whom to test" for COVID-19 has been prescribed by the Indian Council of Medical Research (ICMR), Department of Health Research (DHR), Ministry of Health and Family Welfare (MOHFW), Government of India. The strategy, which has been timely revised on frequent intervals, with the latest revision on May 18, 2020 (version 5), includes testing of:

1. All symptomatic (influenza-like illness [ILI] symptoms, i.e., acute respiratory infection with fever and cough) individuals with a history of international travel in the last 14 days.

2. All symptomatic (ILI symptoms) contacts of laboratoryconfirmed cases.

3. All symptomatic (ILI symptoms) healthcare workers/ frontline workers involved in containment and mitigation of COVID-19.

4. All patients of severe acute respiratory infection (SARI), that is, acute respiratory infection with fever and cough, requiring hospitalization.

5. Asymptomatic direct and high-risk contacts of a confirmed case to be tested once between day 5 and day 10 of coming into contact.

6. All symptomatic ILI within hotspots/containment zones, and among returnees and migrants within 7 days of illness.

7. All hospitalized patients who develop ILI symptoms.

8. Pregnant women residing in clusters/containment areas or large migration gatherings/evacuee centers from hotspot districts, presenting in labor or likely to deliver in the next 5 days, should be tested even if asymptomatic.

No emergency procedure (including deliveries) should be delayed for lack of test. ${ }^{10}$ However, the Infectious Disease Society of America (IDSA) advised that SARS-COV-2 RNA testing should also be performed in asymptomatic persons undergoing major time-sensitive surgeries or aerosolgenerating procedures, or who are immunocompromised, or before the immunosuppressive procedure. ${ }^{11}$

\section{Preparedness of Molecular Laboratory for COVID-19}

As of May 19, 2020, 391 Government laboratories and 164 Private laboratories are assigned for testing. Real-time reverse transcriptase polymerase chain reaction (rRT-PCR), TrueNat test, and CBNAAT test for COVID-19 are being conducted in 431, 77 and 47 laboratories, respectively. AIIMS Jodhpur also has a regional-level viral research and diagnostic laboratory (R-VRDL), mentoring 38 government and private laboratories for diagnosis of COVID-19 in the states of Rajasthan and Gujarat. ${ }^{12}$

COVID-19 testing laboratories should have a designated area for sample collection of suspected patients, and dedicated rooms for RNA extraction, reagents preparation and performing rRT-PCR. The laboratory should have a minimum BSL-II facility equipped with Class II A2 biosafety cabinets, laminar flow cabinets, RT-PCR machine, cold centrifuge, vortex mixer, freezer of $-80^{\circ} \mathrm{C},-20^{\circ} \mathrm{C}$ and $4^{\circ} \mathrm{C}$, and provision for data recording and keeping. The consumables required for sample collection are viral transport media (VTM), dacron or polyester swab sticks, etc., along with reagents and consumables for extraction of RNA (e.g., RNA extraction kit) and 
nucleic acid amplification test (NAAT) (e.g., rRT-PCR kit). The strict precautions for biomedical waste (BMW) disposal have to be followed as per MOHFW/Central Pollution Control Board (CPCB) guidelines for COVID-19 BMW management. ${ }^{13,14}$

Molecular diagnosis of COVID-19: Appropriate specimen collection with prompt and accurate testing of patients, meeting the suspected case definition for COVID-19, is a priority for clinical management and outbreak control. ${ }^{15}$

\section{Choice of Molecular Test for Detection of SARS-CoV-2}

The diagnosis of SARS-CoV2 started with random amplification deep sequencing molecular methods such as next-generation sequencing (NGS). ${ }^{16}$ Routine confirmation of cases of COVID-19 is based on the detection of unique sequences of viral RNA by NAAT. The most widely used NAAT is rRT-PCR followed by isothermal amplification methods. ${ }^{17} \mathrm{~A}$ major advantage of rRT-PCR assay is that amplification and analysis are done simultaneously in a closed system to minimize product contamination. Viral cultures are not recommended for routine diagnosis..$^{16}$ Rapid point-of-care tests comprising antigen and antibody detection methods are still unsatisfactory and are used only for epidemiological studies. ${ }^{17}$

\section{Choice of Specimen, Transportation, and Storage of Specimen for COVID-19 Testing}

The most preferred specimens are as follows:

1. Upper respiratory tract (URT) specimens: nasopharyngeal swab (NPS), oropharyngeal swab (OPS), nasal midturbinate swab, anterior nares (nasal swab), nasopharyngeal wash/aspirate, or nasal wash/aspirate in ambulatory patients.

2. Lower respiratory specimens: sputum (if produced) and/ or endotracheal aspirate or bronchoalveolar lavage (BAL) in patients with more severe respiratory disease, ${ }^{15,18}$ and if PCR is negative on URT sample inpatient of high-index of suspicion. ${ }^{11}$

Few studies have shown that SARS-CoV-2 RNA was detected by rRT-PCR in $93 \%$ of BAL, $72 \%$ of sputum, but only in $32 \%$ of OPS, which was significantly lower than that in NPS (63\%). ${ }^{19}$ Hence, NPS are preferred over OPS or collected along with OPS in the same VTM tube. ${ }^{18}$ Samples should be collected by Dacron or polyester flocked swabs and transported in VTM containing antifungal and antibiotic supplements in ice packs or maintaining the cold chain. If any delay is anticipated while transportation, specimen should be stored at 2 to $8^{\circ} \mathrm{C}$ if $\leq 5$ days and at $-20^{\circ} \mathrm{C}$ to $-80^{\circ} \mathrm{C}$ if stored for a longer duration. ${ }^{15,18}$

Processing of Sample: Clinical samples/swabs collected in VTM are processed and viral RNA is extracted and purified either by manually using various authorized RNA extraction kits or by fully automated closed systems. This is followed by target amplification and detection by various methods.

\section{Nucleic Acid Extraction}

Commercially available nucleic acid extraction kits can be used to extract RNA of SARS-CoV-2. The kit contains a lysis buffer, which is composed of a guanidinium-based inactivating agent as well as a nondenaturing detergent, that lyses the envelope of an RNA virus and inactivates RNases. It is followed by washing with wash buffer and extracting or eluting of the virus containing nucleic acids.

RNA extraction kits commercially available for COVID-19 are as follows:

1. Manual nucleic acid extraction kits-

a. QIAamp viral RNAMiniKits-Spin column-based.

b. MGI easy nucleic acid extraction kit-Magnetic beadbased.

c. Maverick magnetic bead-based nucleic acid extraction kit-Magnetic bead-based.

d. TRUPCR COVID-19 viral RNA extraction kit-Spin column-based.

2. Automated nucleic acid extraction methods, for example, Qiagen Automated RNA extraction by QIACUBE-micro spin-based technology.

\section{Real-time RT-PCR for COVID-19}

ICMR and WHO recommend rRT-PCR as the molecular test of choice for COVID-19 diagnosis. The real-time assay uses the TaqMan fluorogenic probe-based chemistry which, in turn, employs the 5'-nuclease activity of Taq DNA polymerase and enables the detection of a specific PCR product as it accumulates during PCR cycles. To perform the assay, the viral RNA is extracted and added to a master mix. The master mix contains nuclease-free water, forward and reverse primers, fl uorophore-quencher probe, and reaction mix (consisting of reverse transcriptase, polymerase, magnesium, nucleotides, and additives). The master mix and extracted RNA are loaded into a PCR thermocycler, and the recommended cycling temperature conditions are set to run the assay. During rRT-PCR, the fluorophore-quencher probe is cleaved, generating a fluorescent signal. The fluorescent signal is detected by the thermocycler, and the amplification progress is recorded in real-time.

Several RT-PCR protocols for detection of SARS-CoV-2 RNA have been advocated by the WHO and ICMR ${ }^{20}$

\section{Target Selection for Molecular Assays}

The most commonly used target genes for diagnosis, which have conserved sequences are as follows:

1. RdRp gene.

2. Open reading frames ORF1 $\mathrm{a}$ and ORF1b (HKU-Orf1 abgene).

3. E gene (envelope protein gene).

4. $\mathrm{N}$ gene (nucleocapsid protein gene).

Other target genes are S gene (spike glycoprotein), transmembrane (M), helicase (Hel), and hemagglutinin-esterase (HE) gene. ${ }^{15,16}$ 
To avoid potential cross-reaction with other endemic coronaviruses as well as potential effects of genetic drift of SARS$\mathrm{CoV}-2$, especially as the virus evolves within new populations, at least two molecular targets should be included in the assay: a broad range of SARS-like Coronaviruses (subgenus Sarbecovirus), inclusive of SARS-CoV-2 (e.g., E gene), along with a specific target for SARS-CoV-2 (e.g., RdRp gene). ${ }^{16,21}$

\section{Quality Control Assay and Interpretation of rRT-PCR Result}

The PCR procedure should include appropriate negative template control (NTC) (one for extraction procedure and one for PCR run), positive template control (PTC), and internal extraction control (IEC)/human housekeeping gene target control (e.g., RNaseP), which should be first validated in each run. NTC should show no fluorescence growth curves crossing the threshold line. IEC should exhibit reaction curves that cross the threshold line at or before 35 cycles, which denotes the presence of sufficient RNA from human tissue and thus an acceptable quality sample. PTC should produce a positive result between 20 and 35 cycles..$^{17,20}$

Failure to detect RNase $\mathrm{P}$ in any of the clinical samples may indicate:

a) Absence of sufficient human cellular material in the sample to enable detection, that is, unacceptable/poor quality sample.

b) Improper extraction of nucleic acid from clinical materials.

c) Carryover of PCR inhibitors from clinical specimens.

d) Improper assay set up and execution.

e) Reagent or equipment malfunction.

If all these criteria are met, then the test sample is interpreted for the sigmoid reaction curve and cycle threshold $(\mathrm{Ct})$ value between 20 and 35 cycles. ${ }^{17,20}$

\section{Biosafety Practices in the Laboratory}

Processing of clinical/respiratory specimens for molecular testing should be done in a BSL-2 or equivalent facilities. Appropriate disinfectants (e.g., hypochlorite [bleach], alcohol, hydrogen peroxide, quaternary ammonium compounds, and phenolic compounds) should be used for the recommended contact time at the correct dilution. Appropriate personal protective equipment (PPE) should be worn by all laboratory personnel handling these specimens. ${ }^{22}$

\section{Isothermal Nucleic Acid Amplification-based Methods}

These techniques are conducted at a single temperature and do not need specialized laboratory equipment. These techniques include recombinase polymerase amplification, transcription-mediated amplification (TMA), nucleic acid sequence-based amplification (NASBA), strand displacement amplification (SDA), helicase-dependent amplification (HDA), and loop-mediated isothermal amplification (LAMP) ${ }^{23,24}$

\section{LAMP-based Methods}

LAMP is a novel isothermal nucleic acid test (NAT). RT-LAMP uses four to six primers to bind to six distinct regions on the target genome. Among the four primers, two are inner primers (forward and reverse) and two are outer primers. LAMP is highly specific. Amplified DNA can be detected as the turbidity due to precipitation of magnesium pyrophosphate, as color (addition of a pH-sensitive dye), or as fluorescence (due to the use of a fluorescent dye that binds to double-stranded DNA). ${ }^{23,24}$ Several LAMP-based CoV detection methods are used in the clinical diagnosis of COVID-19..$^{25-28}$

\section{Commercial Molecular Assay Kits}

Various NAAT kits have been granted in vitro diagnostic emergency use authorization (EUA) by the US Food and Drugs Administration (FDA), ${ }^{29} \mathrm{WHO}^{30}$ and ICMR $^{31}$ and Foundation for Innovative New Diagnostics (FIND) $)^{32}$ as enlisted in - Tables 1-4.

\section{Emerging Molecular Techniques for COVID-19}

\section{Detection of COVID-19 using CRISPR/Cas12a}

A CRISPR/Cas12a-based rapid nucleic acid detection assay with a naked eye readout, CRISPR/Cas12a-NER includes Cas12a protein, SARS-CoV-2 specific CRISPR RNAs (crRNAs), and a single-stranded DNA (ssDNA) reporter. It can detect as few as 10 copies of the virus gene in 45 minutes. It gives comparable results as qPCR assay, and it is a simple and reliable on-site diagnostic method suitable for a local hospital or community testing. Reverse transcript recombinase-aided amplification (RT-RAA) is used to obtain enough DNA for Cas12a-mediated detection. The RT-RAA will amplify the target gene fragment in 30 minutes at $39^{\circ} \mathrm{C}$, followed by a CRISPR/Cas12a reaction at $37^{\circ} \mathrm{C}$ for 15 minutes. SARS-CoV-2 genome is detected by targeting a total of 14 crRNAs, designed on four domains of the orf1a, orf1 b, N, and E genes. To enable onsite diagnosis, an ssDNA reporter labeled with a quenched green fluorescent molecule is used which is cleaved by Cas12a; when there is nucleic acid of SARS-CoV-2, it results in green fluorescence under $485 \mathrm{~nm}$ light. ${ }^{33,34}$

\section{Sherlock CRISPR SARS-CoV-2 Kit}

Specific high-sensitivity enzymatic reporter unlocking (SHERLOCK) allows multiplexed, portable, and ultrasensitive detection of RNA or DNA from clinically relevant samples with recombinase-mediated polymerase preamplification of DNA or RNA and subsequent Cas13- or Cas12-mediated detection via fluorescence and colorimetric readouts that provide results in $<1$ hour with a setup time of less than 15 minutes. The SARS-CoV-2 nucleic acid is first extracted, isolated, and purified. The purified nucleic acid is then subjected to RT-LAMP where targeted SARS-CoV-2 genomic RNA is reverse transcribed to DNA, and this DNA is amplified by a strand, displacing DNA polymerase. In a second step, the amplified DNA is 
Table 1 EUA by US FDA tests, based on manual rRT-PCR (as on May 21, 2020)

\begin{tabular}{|c|c|c|c|c|}
\hline \multicolumn{5}{|c|}{ Tests based on manual rRT-PCR (all qualitative) } \\
\hline S. no & Company name & Kit name & Target genes & $\begin{array}{l}\text { Special } \\
\text { remarks }\end{array}$ \\
\hline 1. & BGI Genomics Co. Ltd. & $\begin{array}{l}\text { Real-time fluorescent RT-PCR kit for detecting SARS-CoV-2 } \\
\text { https://www.fda.gov/media/136472/download }\end{array}$ & $\begin{array}{l}\text { ORF1ab gene, human } \\
\beta \text {-actin (IC) }\end{array}$ & $\begin{array}{l}\text { EUA by } \\
\text { WHO and } \\
\text { ICMR too }\end{array}$ \\
\hline 2. & PerkinElmer, Inc. & $\begin{array}{l}\text { PerkinElmer new Coronavirus nucleic acid detection kit } \\
\text { https://www.fda.gov/media/136410/download }\end{array}$ & ORF1ab and $\mathrm{N}$ genes & \multirow[t]{2}{*}{$\begin{array}{l}\text { EUA by } \\
\text { WHO' too }\end{array}$} \\
\hline 3. & Primerdesign Ltd. & $\begin{array}{l}\text { Primerdesign Ltd COVID-19 genesig real-time PCR assay } \\
\text { https://www.fda.gov/media/136309/download }\end{array}$ & & \\
\hline 4. & $\begin{array}{l}\text { Thermo Fisher } \\
\text { Scientific, Inc. }\end{array}$ & $\begin{array}{l}\text { TaqPath COVID-19 combo kit } \\
\text { https://www.fda.gov/media/136112/download }\end{array}$ & $\begin{array}{l}\text { Orf-1ab gene, } \mathrm{N} \text { gene, } \\
\text { S MS2 phage (IC) }\end{array}$ & \multirow{4}{*}{$\begin{array}{l}\text { EUA by } \\
\text { WHO }^{\mathrm{b}} \\
\text { (under } \\
\text { process) } \\
\text { too }\end{array}$} \\
\hline 5. & $\begin{array}{l}\text { InBios International, } \\
\text { Inc. }\end{array}$ & $\begin{array}{l}\text { Smart Detect SARS-CoV-2 rRT-PCR Kit } \\
\text { https://www.fda.gov/media/136786/download }\end{array}$ & $\begin{array}{l}\text { Orf1ab gene, E gene, } \\
\text { Ngene RNase P (IC) }\end{array}$ & \\
\hline 6. & SansureBioTech Inc. & $\begin{array}{l}\text { Novel Coronavirus (2019-nCoV) nucleic acid diagnostic } \\
\text { kit (PCR-Fluorescence Probing) } \\
\text { https://www.fda.gov/media/137651/download }\end{array}$ & $\begin{array}{l}\text { Orf1ab gene, } \mathrm{N} \text { gene } \\
\text { RNase P (IC) }\end{array}$ & \\
\hline 7. & Fast Track Diagnostics & $\begin{array}{l}\text { FTD SARS-CoV-2 } \\
\text { https://www.fda.gov/media/137690/download }\end{array}$ & $\begin{array}{l}\text { ORF1ab gene Ngene } \\
\text { Equine arteritis virus (IC) }\end{array}$ & \\
\hline 8. & SD Biosensor, Inc. & $\begin{array}{l}\text { STANDARD M nCoV real-time detection kit } \\
\text { https://www.fda.gov/media/137302/download }\end{array}$ & $\begin{array}{l}\text { ORF1ab gene, RdRp gene, } \\
\text { E gene pseudovirus con- } \\
\text { taining RNA target (IC) }\end{array}$ & \multirow[t]{3}{*}{$\begin{array}{l}\text { EUA by } \\
\text { ICMR too }\end{array}$} \\
\hline 9. & $\begin{array}{l}\text { Altona Diagnostics } \\
\text { GmbH }\end{array}$ & $\begin{array}{l}\text { RealStar SARS-CoV02 RT-PCR kits U.S. } \\
\text { https://www.fda.gov/media/137252/download }\end{array}$ & $\begin{array}{l}\text { E gene } S \text { gene } \\
\text { artificial RNA template (IC) }\end{array}$ & \\
\hline 10. & Seegene, Inc. & $\begin{array}{l}\text { Allplex 2019-nCoV assay } \\
\text { https://www.fda.gov/media/137178/download }\end{array}$ & $\begin{array}{l}\text { RdRp gene, E gene, } \mathrm{N} \text { gene } \\
\text { MS2 phage (IC) }\end{array}$ & \\
\hline 11. & OSANG Healthcare & $\begin{array}{l}\text { GeneFinder COVID-19 Plus RealAmp Kit } \\
\text { https://www.fda.gov/media/137116/download }\end{array}$ & $\begin{array}{l}\text { RdRp gene, E gene, } N \text { gene } \\
\text { RNaseP (IC) }\end{array}$ & \\
\hline 12. & $\mathrm{CDC}$ & $\begin{array}{l}\text { CDC 2019-novel Coronavirus (2019-nCoV) real-time } \\
\text { RT-PCR } \\
\text { diagnostic panel (reiussed 03/15/2020) } \\
\text { https://www.fda.gov/media/134922/download }\end{array}$ & $\begin{array}{l}\text { Two primer sets (N1 and } \\
\text { N2) for } \mathrm{N} \text { gene RNaseP (IC) }\end{array}$ & \\
\hline 13. & $\begin{array}{l}\text { Wadsworth Center, } \\
\text { NYSDOH }\end{array}$ & $\begin{array}{l}\text { New York SARS-CoV-2 real-time reverse transcriptase } \\
\text { (RT)-PCR diagnostic panel (reissued 03/10/2020) } \\
\text { https://www.fda.gov/media/135847/download }\end{array}$ & Ngene RNaseP (IC) & \\
\hline 14. & $\begin{array}{l}\text { Laboratory Corporation } \\
\text { of America }\end{array}$ & $\begin{array}{l}\text { COVID-19 RT-PCR test (reissued April 20, 2020) } \\
\text { https://www.fda.gov/media/136151/download }\end{array}$ & RNaseP (IC) & \\
\hline 15. & $\begin{array}{l}\text { Quest Diagnostics } \\
\text { Infectious Disease, Inc. }\end{array}$ & $\begin{array}{l}\text { Quest SARS-CoV-2 rRT-PCR } \\
\text { https://www.fda.gov/media/136231/download }\end{array}$ & $\begin{array}{l}\text { nucleocapsid gene } \\
\text { (N1 \& N3) }\end{array}$ & \\
\hline 16. & Quidel Corp & $\begin{array}{l}\text { Lyra SARS-CoV-2 assay } \\
\text { https://www.fda.gov/media/136820/download }\end{array}$ & $\begin{array}{l}\text { nonstructural Polyprotein } \\
\text { (pp1ab) } \\
\text { MS2 phage (IC) }\end{array}$ & \\
\hline 17. & $\begin{array}{l}\text { Avellino Laboratory } \\
\text { USA, Inc. }\end{array}$ & $\begin{array}{l}\text { AvellinoCoV2 test } \\
\text { https://www.fda.gov/media/136453/download }\end{array}$ & N gene RNaseP $(I C)$ & \\
\hline 18. & Ipsum Diagnostics, LLC & $\begin{array}{l}\text { COV-19 IDx assay } \\
\text { https://www.fda.gov/media/136621/download }\end{array}$ & $N$ gene RNase $P(I C)$ & \\
\hline 19. & $\begin{array}{l}\text { ScienCell Research } \\
\text { Laboratories }\end{array}$ & $\begin{array}{l}\text { ScienCell SARS-CoV-2 Coronavirus real-time RT-PCR } \\
\text { (RT- qPCR) detection kit } \\
\text { https://www.fda.gov/media/136691/download }\end{array}$ & $\begin{array}{l}\text { N gene }(\mathrm{N} 1, \mathrm{~N} 2) \text { RNase } \\
\mathrm{P}(\mathrm{IC})\end{array}$ & \\
\hline 20. & Gnomegen LLC & $\begin{array}{l}\text { Gnomegen COVID-19 RT-digital PCR detection kit } \\
\text { https://www.fda.gov/media/136738/download }\end{array}$ & $\mathrm{N}$ gene RNase $\mathrm{P}(\mathrm{IC})$ & \\
\hline 21. & DiaCarta, Inc. & $\begin{array}{l}\text { QuantiVirus SARS-CoV-2 test kit } \\
\text { https://www.fda.gov/media/136806/download }\end{array}$ & $\begin{array}{l}\text { Orf1ab gene, E gene, } \\
\text { N gene RNaseP(IC) }\end{array}$ & \\
\hline 22. & $\begin{array}{l}\text { Becton, Dickinson \& } \\
\text { Company }\end{array}$ & $\begin{array}{l}\text { BD SARS-CoV-2 reagents for BD MAX System } \\
\text { https://www.fda.gov/media/136816/download }\end{array}$ & $\begin{array}{l}\mathrm{N} \text { gene (N1, N2) } \\
\text { RNaseP (IC) }\end{array}$ & \\
\hline 23. & $\begin{array}{l}\text { Maccura Biotechnology } \\
\text { (USA) LLC }\end{array}$ & $\begin{array}{l}\text { SARS-CoV-2 fluorescent PCR Kit } \\
\text { https://www.fda.gov/media/137026/download }\end{array}$ & $\begin{array}{l}\text { Orf1ab gene, E gene, } \\
\mathrm{N} \text { gene MS2 phage (IC) }\end{array}$ & \\
\hline
\end{tabular}


Table 1 (Continued)

\begin{tabular}{|c|c|c|c|c|}
\hline \multicolumn{5}{|c|}{ Tests based on manual rRT-PCR (all qualitative) } \\
\hline S. no & Company name & Kit name & Target genes & $\begin{array}{l}\text { Special } \\
\text { remarks }\end{array}$ \\
\hline 24. & KorvaLabs Inc. & $\begin{array}{l}\text { Curative-Korva SARS-Cov-2 assay } \\
\text { https://www.fda.gov/media/137089/download }\end{array}$ & $\begin{array}{l}\mathrm{N} \text { gene }(\mathrm{N} 1, \mathrm{~N} 2) \\
\text { RNaseP (IC) }\end{array}$ & \\
\hline 25. & GenoSensor, LLC & $\begin{array}{l}\text { GS COVID-19 RT-PCR kit } \\
\text { https://www.fda.gov/media/137093/download }\end{array}$ & $\begin{array}{l}\text { Orf1ab gene, E gene, } \\
\text { N gene GUSB gene (IC) }\end{array}$ & \\
\hline 26. & Fosun Pharma USA Inc. & $\begin{array}{l}\text { Fosun COVID-19 RT-PCR detection kit } \\
\text { https://www.fda.gov/media/137120/download }\end{array}$ & $\begin{array}{l}\text { Orf1ab gene, E gene, } \\
\mathrm{N} \text { gene Lentivirus (IC) }\end{array}$ & \\
\hline 27. & $\begin{array}{l}\text { Trax Management } \\
\text { Services Inc }\end{array}$ & $\begin{array}{l}\text { PhoenixDx 2019-CoV } \\
\text { https://www.fda.gov/media/137153/download }\end{array}$ & $\begin{array}{l}\text { RdRp gene and E gene } \\
\text { RNaseP (IC) }\end{array}$ & \\
\hline 28. & SEASUN BIOMATERIALS & $\begin{array}{l}\text { U-TOP COVID-19 detection kit } \\
\text { https://www.fda.gov/media/137425/download }\end{array}$ & $\begin{array}{l}\text { Orf1ab gene, } \mathrm{N} \text { gene } \\
\text { RNaseP (IC) }\end{array}$ & \\
\hline 29. & LabGenomics Co., Ltd. & $\begin{array}{l}\text { LabGun COVID-19 RT-PCR kit } \\
\text { https://www.fda.gov/media/137483/download }\end{array}$ & $\begin{array}{l}\text { RdRp gene and E gene } \\
\text { MS2 phage (IC) }\end{array}$ & \\
\hline 30. & BioMérieux SA & $\begin{array}{l}\text { SARS-COV-2 R-GENE } \\
\text { https://www.fda.gov/media/137742/download }\end{array}$ & $\begin{array}{l}\text { RdRp gene, } \mathrm{N} \text { gene and } \\
\text { E gene }\end{array}$ & \\
\hline 31. & $\begin{array}{l}\text { OPTI Medical Systems, } \\
\text { Inc. }\end{array}$ & $\begin{array}{l}\text { OPTI SARS-CoV-2 RT PCR test } \\
\text { https://www.fda.gov/media/137739/download }\end{array}$ & $\begin{array}{l}\mathrm{N} \text { gene }(\mathrm{N} 1, \mathrm{~N} 2) \text { RNase } \\
\mathrm{P}(\mathrm{IC})\end{array}$ & \\
\hline 32. & $\begin{array}{l}\text { RutgersClinical } \\
\text { Genomics } \\
\text { Laboratory }\end{array}$ & $\begin{array}{l}\text { Rutgers Clinical Genomics Laboratory TaqPath } \\
\text { SARS-CoV-2-assay } \\
\text { https://www.fda.gov/media/136875/download }\end{array}$ & $\begin{array}{l}\text { Orf1ab gene, S gene, } \\
\text { N gene MS2 Phage (IC) }\end{array}$ & $\begin{array}{l}\text { EUA for } \\
\text { saliva too }\end{array}$ \\
\hline 33. & $\begin{array}{l}\text { Zymo Research } \\
\text { Corporation }\end{array}$ & $\begin{array}{l}\text { Quick SARS-CoV-2rRT-PCR kit } \\
\text { https://www.fda.gov/media/137780/download }\end{array}$ & $\mathrm{N}$ gene RNaseP (IC) & \\
\hline 34. & 1drop Inc. & $\begin{array}{l}\text { One copy COVID-19 qPCR multikit } \\
\text { https://www.fda.gov/media/137935/download }\end{array}$ & $\begin{array}{l}\text { RdRp gene and E gene } \\
\text { human GAPDH mRNA (IC) }\end{array}$ & \\
\hline 35. & $\begin{array}{l}\text { Applied DNA Sciences, } \\
\text { Inc }\end{array}$ & $\begin{array}{l}\text { LineaCOVID-19 assay kit https://www.fda.gov/ } \\
\text { media/138059/download }\end{array}$ & S gene RNase $P(I C)$ & \\
\hline 36. & GeneMatrix, Inc. & $\begin{array}{l}\text { NeoPlex COVID-19 detection kit } \\
\text { https://www.fda.gov/media/138101/download }\end{array}$ & RNase $\mathrm{P}(\mathrm{IC})$ & $\begin{array}{l}\text { EUA by } \\
\text { ICMR too }\end{array}$ \\
\hline 37. & $\begin{array}{l}\text { Assurance Scientific } \\
\text { Laboratories }\end{array}$ & $\begin{array}{l}\text { Assurance SARS-CoV-2 panel } \\
\text { https://www.fda.gov/media/138154/download }\end{array}$ & $\begin{array}{l}\mathrm{N} \text { gene }(\mathrm{N} 1, \mathrm{~N} 2) \\
\text { RNase } \mathrm{P}(\mathrm{IC})\end{array}$ & \\
\hline 38. & $\begin{array}{l}\text { Fulgent Therapeutics, } \\
\text { LLC }\end{array}$ & $\begin{array}{l}\text { Fulgent COVID-19 by RT-PCR test } \\
\text { https://www.fda.gov/media/138150/download }\end{array}$ & $\begin{array}{l}\mathrm{N} \text { gene }(\mathrm{N} 1, \mathrm{~N} 2) \text { RNase } \\
\mathrm{P}(\mathrm{IC})\end{array}$ & \\
\hline 39. & Quidel Corporation & $\begin{array}{l}\text { Lyra direct SARS-CoV-2 assay } \\
\text { https://www.fda.gov/media/138179/download }\end{array}$ & $\begin{array}{l}\text { Nonstructural Polyprotein } \\
\text { (pp1ab) gene MS2 Phage } \\
\text { (IC) }\end{array}$ & \\
\hline
\end{tabular}

Abbreviations: EUA, emergency use authorization; FDA, Food and Drug Administration; rRT-PCR, real-time reverse transcription polymerase chain reaction, IC- Internal Control.

aWHO emergency use listing for in vitro diagnostics (IVDs) detecting SARS-CoV-2 nucleic acid (last update: May 21, 2020).

bSARS-CoV-2 nucleic acid tests: progress of the active applications in the emergency use listing assessment pipeline

(last update: May 21, 2020).

transcribed to activate the collateral cleavage activity of a CRISPR complex programmed to the target RNA sequence. This collateral activity results in cleavage of nucleic acid reporters, resulting in a fluorescent readout detected by a plate reader. ${ }^{23,24,29,35}$

\section{Point-of-care Molecular Assays}

\section{Cepheid Xpert Xpress SARS-CoV-2 Test}

The Xpert 94 Xpress SARS-CoV-2 test (Cepheid) uses the GeneXpert Dx System which performs automated specimen processing, RNA extraction, rRT-PCR of SARS-CoV-2 RNA, and amplicon detection in a single cartridge. The test detects nucleocapsid gene (N2) and envelope gene (E) (EUA version) with additional RdRp genes (research used only [RUO] version], and results are generated in approximately 45 minutes. Using $\mathrm{E}$ gene, $\mathrm{N}$ gene, sample processing control (SPC), and probe check control, the results are interpreted as the positive, presumptive, or negative. The limit of detection of the Xpert test was 0.01 plaque-forming units (PFU)/mL. Compared with standard of care (SOC) NAATs, the positive agreement of the Xpert test was 99.5\% and the negative agreement was $95.8 \%$. ICMR recommends using Cepheid Xpert Xpress SARS CoV-2 for diagnosis of COVID-19. Currently, 47 CBNAAT laboratories are working all over India for COVID-19 diagnosis. ${ }^{21,29}$ 
Table 2 EUA by US FDA tests, based on automated rRT-PCR (as on May 21, 2020)

\begin{tabular}{|c|c|c|c|c|c|}
\hline \multicolumn{6}{|c|}{ Tests based on automated rRT-PCR (all qualitative) } \\
\hline S.NO. & Company name & Kit name & Test principle & Target gene & Remarks \\
\hline 1. & $\begin{array}{l}\text { Roche Molecular } \\
\text { Systems, Inc. } \\
\text { (RMS) }\end{array}$ & $\begin{array}{l}\text { cobas } \\
\text { SARS-CoV-2 }\end{array}$ & $\begin{array}{l}\text { rRT-PCR using fully automated Cobas } \\
6800 / 8800 \text { Systems https://www.fda.gov/ } \\
\text { media/136049/download }\end{array}$ & $\begin{array}{l}\text { orf1gene and } \\
\text { E-gene }\end{array}$ & $\begin{array}{l}\text { EUA by } \\
\text { WHO }^{\text {a }} \text { too }\end{array}$ \\
\hline 2. & $\begin{array}{l}\text { Abbott } \\
\text { Molecular }\end{array}$ & $\begin{array}{l}\text { Abbott RealTime } \\
\text { SARS- CoV-2 } \\
\text { assay }\end{array}$ & $\begin{array}{l}\text { rRT-PCR using Abbott m2000 System consisting } \\
\text { of sample preparation unit (Abbott m2000sp) and } \\
\text { amplification and detection unit (Abbottm2000rt) } \\
\text { https://www.fda.gov/media/136258/download }\end{array}$ & $\begin{array}{l}\text { RdRp gene, } \\
\text { N gene }\end{array}$ & $\begin{array}{l}\text { EUA by } \\
\text { WHO too }\end{array}$ \\
\hline 3. & $\begin{array}{l}\text { DiaSorin } \\
\text { Molecular LLC }\end{array}$ & $\begin{array}{l}\text { Simplexa COVID- } \\
19 \text { Direct }\end{array}$ & $\begin{array}{l}\text { rRT-PCR using direct amplification disc and fluores- } \\
\text { cent probes https://www.fda.gov/media/136286/ } \\
\text { download }\end{array}$ & $\begin{array}{l}\text { Orf1ab gene, } \\
\text { S gene }\end{array}$ & $\begin{array}{l}\text { EUA by } \mathbf{W H O}^{\mathrm{b}} \\
\text { (under } \\
\text { process) too }\end{array}$ \\
\hline 4. & $\begin{array}{l}\text { BioFire } \\
\text { Diagnostics, LLC }\end{array}$ & $\begin{array}{l}\text { BioFire Respiratory } \\
\text { Panel } 2.1 \text { (RP2.1) }\end{array}$ & $\begin{array}{l}\text { Nested multiplex rRT-PCR in the closed cartridge } \\
\text { system } \\
\text { https://www.fda.gov/media/137580/download }\end{array}$ & \multirow{2}{*}{$\begin{array}{l}\text { RNA transcript } \\
\text { from yeast Schizo } \\
\text { saccharomyces } \\
\text { pombe (IC) }\end{array}$} & \\
\hline 5. & $\begin{array}{l}\text { BioFireDefense, } \\
\text { LLC }\end{array}$ & $\begin{array}{l}\text { BioFire COVID-19 } \\
\text { Test }\end{array}$ & $\begin{array}{l}\text { Nested multiplex rRT-PCR using a fully automated } \\
\text { cartridge-based system } \\
\text { https://www.fda.gov/media/136356/download }\end{array}$ & & \\
\hline 6. & $\begin{array}{l}\text { Luminex } \\
\text { Corporation }\end{array}$ & $\begin{array}{l}\text { ARIES SARS-CoV-2 } \\
\text { Assay }\end{array}$ & $\begin{array}{l}\text { rRT-PCR in the closed cassette system } \\
\text { https://www.fda.gov/media/136693/download }\end{array}$ & $\begin{array}{l}\text { orf1ab gene, } \mathrm{N} \\
\text { gene and } \mathrm{E} \text { gene }\end{array}$ & \\
\hline 7. & Hologic, Inc. & $\begin{array}{l}\text { Panther Fusion } \\
\text { SARS- CoV-2 Assay }\end{array}$ & $\begin{array}{l}\text { rRT-PCR using a fully automated cartridge-based } \\
\text { system https://www.fda.gov/media/136156/ } \\
\text { download }\end{array}$ & $\begin{array}{l}\text { ORF1ab gene } \\
\text { Region } 1 \\
\text { and } 2\end{array}$ & \\
\hline 8. & $\begin{array}{l}\text { Becton, } \\
\text { Dickinson \& } \\
\text { Company (BD) }\end{array}$ & $\begin{array}{l}\text { BioGX SARS-CoV-2 } \\
\text { Reagents for BD } \\
\text { MAX System }\end{array}$ & $\begin{array}{l}\text { rRT-PCR using closed system (multiplexed } \\
\text { detection of N1 and RNase P \& N2 and } \\
\text { RNase P) https://www.fda.gov/media/136650/ } \\
\text { download }\end{array}$ & $\begin{array}{l}\mathrm{N} 1 \text { and } \mathrm{N} 2 \text { gene } \\
\text { RNaseP }(\mathrm{IC})\end{array}$ & \\
\hline 9. & $\begin{array}{l}\text { NeuMoDx } \\
\text { Molecular, Inc. }\end{array}$ & $\begin{array}{l}\text { NeuMoDx } \\
\text { SARS-CoV-2 } \\
\text { Assay }\end{array}$ & $\begin{array}{l}\text { Fully automated sample preparation (nucleic acid } \\
\text { extraction and purification) followed by rRT-PCR } \\
\text { https://www.fda.gov/media/136565/download }\end{array}$ & $\begin{array}{l}\text { Nonstructural } \\
\text { protein } 2 \text { (Nsp2) } \\
\text { gene and N gene } \\
\text { MS2phage (IC) }\end{array}$ & \\
\hline 10. & QIAGEN GmbH & $\begin{array}{l}\text { QIAstat-Dx } \\
\text { Respiratory } \\
\text { SARS-CoV-2 Panel }\end{array}$ & $\begin{array}{l}\text { Nested multiplex rRT-PCR in the closed } \\
\text { cartridge system https://www.fda.gov/ } \\
\text { media/136571/download }\end{array}$ & $\begin{array}{l}\text { Orf1b gene, RdRp } \\
\text { gene, E gene MS2 } \\
\text { phage (IC) }\end{array}$ & \\
\hline 11, & $\begin{array}{l}\text { Abbott } \\
\text { Molecular Inc }\end{array}$ & $\begin{array}{l}\text { Alinity m } \\
\text { SARS-CoV-2 assay }\end{array}$ & $\begin{array}{l}\text { Fully automated sample preparation (nucleic acid } \\
\text { extraction and purification) followed by rRT-PCR } \\
\text { https://www.fda.gov/media/137979/download }\end{array}$ & $\begin{array}{l}\text { RdRp gene, } N \\
\text { gene }\end{array}$ & \\
\hline \multicolumn{6}{|c|}{ Tests based on automated rRT-PCR with special techniques (all qualitative) } \\
\hline 1. & $\begin{array}{l}\text { GenMark } \\
\text { Diagnostics, Inc. }\end{array}$ & $\begin{array}{l}\text { ePlex SARS-CoV-2 } \\
\text { Test }\end{array}$ & $\begin{array}{l}\text { rRT-PCR using automated cartridge based on } \\
\text { electrowetting and eSensor technology (based on } \\
\text { competitive DNA hybridization and } \\
\text { electrochemical detection) https://www.fda.gov/ } \\
\text { media/136282/download }\end{array}$ & & \\
\hline 2. & $\begin{array}{l}\text { Bio-Rad } \\
\text { Laboratories, Inc. }\end{array}$ & $\begin{array}{l}\text { Bio-Rad } \\
\text { SARS-CoV-2 } \\
\text { ddPCR Test }\end{array}$ & $\begin{array}{l}\text { Digital droplet rRT-PCR https://www.fda.gov/ } \\
\text { media/137579/download }\end{array}$ & $\begin{array}{l}\text { N gene RNaseP } \\
\text { (IC) }\end{array}$ & \\
\hline 3. & $\begin{array}{l}\text { Co-Diagnostics, } \\
\text { Inc. }\end{array}$ & $\begin{array}{l}\text { Logix Smart } \\
\text { Coronavirus } \\
\text { Disease 2019 } \\
\text { (COVID-19) Kit }\end{array}$ & $\begin{array}{l}\text { rRT-PCR and detection using CoPrimer technology } \\
\text { [labeled forward CoPrimer acts as both forward } \\
\text { primer and probe] } \\
\text { https://www.fda.gov/media/136687/download }\end{array}$ & RNaseP (IC) & \\
\hline 4. & Rheonix, Inc. & $\begin{array}{l}\text { Rheonix COVID-19 } \\
\text { MDx Assay }\end{array}$ & $\begin{array}{l}\text { rRT-PCR followed by microarray https://www.fda. } \\
\text { gov/media/137489/download }\end{array}$ & $\begin{array}{l}\text { N gene RNaseP } \\
\text { (IC) }\end{array}$ & \\
\hline 5. & $\begin{array}{l}\text { Luminex } \\
\text { Molecular } \\
\text { Diagnostics, Inc. }\end{array}$ & $\begin{array}{l}\text { NxTAGCoV } \\
\text { Extended Panel } \\
\text { Assay }\end{array}$ & $\begin{array}{l}\text { bead rRT-PCR followed by bead hybridization } \\
\text { https://www.fda.gov/media/136500/download }\end{array}$ & $\begin{array}{l}\text { orf1ab gene, } \\
\mathrm{N} \text { gene and } \mathrm{E} \text { gene } \\
\text { MS2 phage (IC) }\end{array}$ & \\
\hline 6. & $\begin{array}{l}\text { Mesa Biotech } \\
\text { Inc. }\end{array}$ & $\begin{array}{l}\text { Accula } \\
\text { SARS-Cov-2 Test }\end{array}$ & $\begin{array}{l}\text { Visual detection by fully automated cassette } \\
\text { system by rRT-PCR https://www.fda.gov/ } \\
\text { media/136355/download }\end{array}$ & RNA phage (IC) & \\
\hline
\end{tabular}

Abbreviations: EUA, emergency use authorization; FDA, Food and Drug Administration; rRT-PCR, real-time reverse transcription polymerase chain reaction, IC- Internal Contro.

${ }^{a}$ WHO emergency use listing for in vitro diagnostics (IVDs) detecting SARS-CoV-2 nucleic acid (last update: May 21, 2020).

'SARS-CoV-2 Nucleic acid tests: progress of the active applications in the emergency use listing assessment pipeline (last update: May 21, 2020). 
Table 3 EUA by US FDA: Tests based on isothermal nucleic acid amplification (all qualitative) (as on May 21, 2020)

\begin{tabular}{|c|c|c|c|c|c|}
\hline 1. & $\begin{array}{l}\text { Sherlock } \\
\text { BioSciences, Inc. }\end{array}$ & $\begin{array}{l}\text { Sherlock } \\
\text { CRISPR } \\
\text { SARS-CoV-2 Kit }\end{array}$ & $\begin{array}{l}\text { RT-LAMP followed by transcription of amplified DNA to acti- } \\
\text { vate a CRISPR complex https://www.fda.gov/media/137746/ } \\
\text { download }\end{array}$ & $\begin{array}{l}\text { ORF1ab gene, } \\
\text { N gene RNase } \\
\text { P (IC) }\end{array}$ & \\
\hline 2. & $\begin{array}{l}\text { Abbott } \\
\text { Diagnostics } \\
\text { Scarborough, Inc. }\end{array}$ & $\begin{array}{l}\text { ID NOW } \\
\text { COVID-19 }\end{array}$ & $\begin{array}{l}\text { Automated isothermal nucleic acid amplification technology } \\
\text { https://www.fda.gov/media/136525/download }\end{array}$ & & $\begin{array}{l}\text { Can be } \\
\text { used in } \\
\text { patient } \\
\text { care } \\
\text { settings }\end{array}$ \\
\hline 3. & Hologic, Inc & $\begin{array}{l}\text { Aptima } \\
\text { SARS- CoV-2 } \\
\text { assay }\end{array}$ & $\begin{array}{l}\text { Automated, and combines the technologies of target cap- } \\
\text { ture, transcription-mediated amplification, and dual kinetic } \\
\text { assay } \\
\text { https://www.fda.gov/media/138096/download }\end{array}$ & ORF1ab gene & \\
\hline 4. & $\begin{array}{l}\text { AtilaBioSyst ems, } \\
\text { Inc. }\end{array}$ & $\begin{array}{l}\text { iAMPCOVID- } \\
19 \text { DetectionKit }\end{array}$ & $\begin{array}{l}\text { rRT isothermal amplification test based on a proprietary } \\
\text { isothermal amplification technology termed OMEGA } \\
\text { amplification }\end{array}$ & $\begin{array}{l}\mathrm{N} \text { gene and } \\
\text { ORF- 1ab gene } \\
\text { human } \\
\text { Gapdhgene } \\
\text { (IC) }\end{array}$ & \\
\hline \multicolumn{6}{|c|}{ (A) Molecular tests that can be used in patient care settings } \\
\hline 1. & Cepheid & $\begin{array}{l}\text { Xpert Xpress } \\
\text { SARS-CoV-2 } \\
\text { test }\end{array}$ & https://www.fda.gov/media/136314/download & & $\begin{array}{l}\text { EUA by } \\
\text { US FDA, } \\
\text { WHO, and } \\
\text { ICMR }\end{array}$ \\
\hline 2. & Mesa Biotech Inc. & $\begin{array}{l}\text { Accula } \\
\text { SARS- Cov-2 } \\
\text { Test }\end{array}$ & $\begin{array}{l}\text { Visual detection by fully automated cassette system by } \\
\text { rRT-PCR } \\
\text { https://www.fda.gov/media/136355/download }\end{array}$ & $\begin{array}{l}\text { RNA phage } \\
\text { (IC) }\end{array}$ & $\begin{array}{l}\text { EUA by US } \\
\text { FDA }\end{array}$ \\
\hline 3. & $\begin{array}{l}\text { Abbott } \\
\text { Diagnostics } \\
\text { Scarborough, } \\
\text { Inc. }\end{array}$ & $\begin{array}{l}\text { ID NOW } \\
\text { COVID-19 }\end{array}$ & $\begin{array}{l}\text { Automated isothermal nucleic acid amplification technology } \\
\text { https://www.fda.gov/media/136525/download }\end{array}$ & & $\begin{array}{l}\text { EUA by } \\
\text { USFDA }\end{array}$ \\
\hline 4. & $\begin{array}{l}\text { Meril Diagnostics } \\
\text { Pvt }\end{array}$ & $\begin{array}{l}\text { Meril COVID-19 } \\
\text { One-step RT- } \\
\text { PCR Kit }\end{array}$ & & & \multirow[t]{2}{*}{$\begin{array}{l}\text { Approved } \\
\text { by DCGI } \\
\text { AND FIND }\end{array}$} \\
\hline 5. & $\begin{array}{l}\text { Molbio } \\
\text { Diagnostics Pvt } \\
\text { Ltd }\end{array}$ & $\begin{array}{l}\text { TrueNAT } \\
\text { SARS CoV-2 }\end{array}$ & & & \\
\hline
\end{tabular}

Abbreviations: EUA, emergency use authorization; DCGI, Drug Controller General of India; FDA, Food and Drug Administration; FIND, Foundation for Innovative New Diagnostics; IC, Internal Control; ICMR, Indian Council for Medical Research; RT-LAMP, reverse transcriptase loop-mediated amplification; rRT-PCR, real-time reverse transcription polymerase chain reaction; WHO, World Health Organization.

${ }^{a}$ WHO emergency use listing for in vitro diagnostics (IVDs) detecting SARS-CoV-2 nucleic acid (last update: May21, 2020).

\section{Truenat betaCoV}

It is a microchip-based rRT-PCR test. As of April 14, 2020, ICMR has validated and recommended Truenat $\beta$ CoV test on Truelab workstation for COVID-19 screening. All positive samples need to be reconfirmed by a separate confirmatory assay for SARS-CoV-2. Throat/nasal swabs will be collected in the VTM used for COVID-19 detection. ${ }^{31}$

\section{ID NOW COVID-19 Test}

It is an automated assay based on isothermal NAT for the qualitative detection of SARS- CoV-2 RNA. It consists of sample receiver and test base which is inserted into the ID NOW Instrument, and the sample is added to the sample receiver where it is transferred via the transfer cartridge to the test base, initiating target amplification. Heating, mixing and detection are provided by the ID NOW Instrument. $^{29}$

\section{Accula SARS-Cov-2 Test}

It is a qualitative, visual detection of nucleic acid from the SARS-CoV-2, using the Accula Dock and Silaris Dock. Test cassette automates NAT, including lysis of the virus, reverse transcriptase of viral RNA to cDNA, nucleic acid amplification, and detection of the SARS- CoV-2 targeted sequences. The test results then are interpreted by the visualization of blue test lines on the detection strip in the test cassette. ${ }^{29}$

\section{5. ePlex SARS-CoV-2Test}

When used with the ePlex instrument, it automates all aspects of NAT including extraction, amplification, and detection, combining electrowetting and GenMark's eSensor technology in a single-use cartridge. eSensor technology is based on the principles of competitive DNA hybridization and electrochemical detection. Electrowetting, or digital microfluidics, uses electrical fields to directly 
Table 4 Molecular assays approved by ICMR (as on May 21, 2020)

\begin{tabular}{|c|c|c|c|}
\hline \multicolumn{4}{|c|}{ Molecular assays approved by ICMR (as on May 21,2020 ) } \\
\hline \multicolumn{3}{|r|}{ Manual NAT } & \multirow{6}{*}{$\begin{array}{l}\text { Approved by } \\
\text { ICMR, DCGI, AND FIND } \\
\text { (https://www. finddx. } \\
\text { org/covid- 19/pipeline/; } \\
\text { updated May 9, 2020] }\end{array}$} \\
\hline 1. & 3B BlackBio Biotech India Ltd & TRUPCR SARS-CoV-2 RT qPCR Kit & \\
\hline \multirow[t]{2}{*}{2.} & Mylab Discovery Solutions Pvt Ltd & PathoDetect CoVID-19 Detection Kit & \\
\hline & \multicolumn{2}{|c|}{ Automated laboratory-based, near-POC NAT, or POC NAT } & \\
\hline 3. & Meril Diagnostics Pvt & Meril COVID-19 One-step RT-PCR kit & \\
\hline 4. & Molbio Diagnostics Pvt Ltd & TrueNAT SARS CoV-2 & \\
\hline 5. & Cepheid & Xpert Xpress SARS-CoV-2 test & \multirow{2}{*}{$\begin{array}{l}\text { EUA by US FDA, WHO } \\
\text { and ICMR }\end{array}$} \\
\hline 6. & BGI Genomics Co. Ltd & Real-time fluorescent RT-PCR kit for detecting SARS-CoV-2 & \\
\hline 7. & SD Biosensor, Inc. & Standard M nCoV real-time detection kit & \multirow{3}{*}{$\begin{array}{l}\text { EUA by US FDA and } \\
\text { ICMR }\end{array}$} \\
\hline 8. & altona Diagnostics GmbH & RealStar SARS-CoV02 RT-PCR kits U.S. & \\
\hline 9. & Seegene, Inc & Allplex 2019-nCoV assay & \\
\hline 10. & Roche Diagnostics, Switzerland & Light Mix Modular SARS-CoV-2 (COVID-19) RdRp & \\
\hline \multicolumn{4}{|c|}{ Others } \\
\hline 11. & Huwel Lifesciences & QuantiplusCoV detection kit ver 2.0 & \multirow[t]{18}{*}{ EUA by ICMR } \\
\hline 12. & ABI (Applied biosystems) & TaqMan 2019-nCoV Control Kit v1 & \\
\hline 13. & Medsource Ozone Biomedicals & COVID-19 RT-PCR kit & \\
\hline 14. & ADT Biotech SdnBhd, Malaysia & LyteStar 2019 nCoV RT-PCR kit 1.0 & \\
\hline 15. & Cosara Diagnostics & SARAGENE Coronavirus (2019 NCV) Test kit & \\
\hline 16. & Labcare Diagnostics & Accucare COVID One-step RT-PCR kit & \\
\hline 17. & POCT services Pvt Ltd & Q-line Molecular Coronavirus (COVID-19) RT-PCR kit & \\
\hline 18. & Helini Biomolecules, Chennai, India & Helini Coronavirus [COVID 19] real-time PCR kit & \\
\hline 19. & Biogenomics (India) & BIO COVID ID/ COVID-19 qualitative PCR detection kit v. 2 & \\
\hline 20. & GCC Biotech, West Bengal, India & DiAGSure nCov-19 detection assay & \\
\hline 21. & $\begin{array}{l}\text { Accelerate Technologies Pte.Itd (DxD } \\
\text { Hub), Singapore }\end{array}$ & A*STAR FORTITUDE KIT 2.0 & \\
\hline 22. & Daan Gene Co. Ltd., China & Daan Gene Co. Ltd & \\
\hline 23. & $\begin{array}{l}\text { Genome Diagnostics Pvt. Ltd., New } \\
\text { Delhi, India }\end{array}$ & GenosensnCOV 2019 real-time PCR kit & \\
\hline 24. & JN Medsys Pte Ltd, Singapore & Protect COVID-19 RT-qPCR kit & \\
\hline 25. & Kogene Biotech, Seoul, Korea & Power Check 2019 nCoV real-time PCR kit & \\
\hline 26. & $\begin{array}{l}\text { Indian Institute of Technology, Delhi, } \\
\text { India }\end{array}$ & COVID-19 probe-free real-time PCR diagnostic kit & \\
\hline 27. & LabGenomics, South Korea & LabGun real-time PCR kit & \\
\hline 28. & OSANG Health Care, South Korea & Gene Finder COVID-19 & \\
\hline
\end{tabular}

Abbreviations: EUA, emergency use authorization; DCGI, Drug Controller General of India; FDA, Food and Drug Administration; FIND, Foundation for Innovative New Diagnostics; IC, Internal Control; ICMR, Indian Council for Medical Research; rRT-PCR, real-time reverse transcription polymerase chain reaction; WHO, World Health Organization.

manipulate discrete droplets on the surface of a hydrophobically coated printed circuit board (PCB). RT-PCR step generates ds-cDNA. Exonuclease digestion creates ss-DNA in preparation for eSensor detection. The amplified targets DNA, and hybridizes to its complementary capture probe and ferrocene-labeled signal probe to form a hybridization complex. Target-specific capture probes are bound to the gold-plated electrodes in the eSensor microarray. The presence of each target is determined by voltammetry which generates specific electrical signals from the ferrocene-labeled signal probe. ${ }^{29}$

\section{Automated Molecular Assays for COVID-19}

\section{Cobas SARS-CoV-2}

Cobas SARS-CoV-2 is an rRT-PCR assay used for the qualitative detection of SARS-CoV-2 RNA in NPS and OPS samples by using Cobas 6800/8800 Systems.

It has fully automated sample preparation (nucleic acid extraction and purification) using magnetic glass particles, followed by PCR amplification and detection. The cobas $6800 / 8800$ systems consist of a sample supply module, transfer module, processing module, and analytic module. It 
targets ORF1/a and E-gene. WHO approved Cobas SARS-CoV-2 assay for diagnosis of COVID-19.29

\section{BioFire Respiratory Panel 2.1 (RP2.1)}

It is a fully automated, multiplexed NAAT intended for simultaneous qualitative detection and differentiation of nucleic acids from multiple respiratory viral and bacterial organisms, including nucleic acid from the SARS-CoV-2 virus. It is used with the FilmArray 2.0 and the FilmArrayTorch Systems. It is based on nested multiplex PCR in a single-use cartridge. ${ }^{29}$

\section{Emerging rRT-PCR Detection Methods}

\section{NxTAGCoV Extended Panel Assay}

The SARS-CoV-2 nucleic acid is first extracted, isolated, and purified, using authorized extraction methods. The purified nucleic acid is then added to preplated, lyophilized bead reagents (LBRs) and mixed to resuspend the reaction reagents. The reaction is amplified via RT-PCR and the reaction product undergoes bead hybridization within the sealed reaction well. The hybridized, tagged beads are then sorted and read on the MAGPIX instrument, or other authorized instruments, and the generated signals analyzed using the NxTAGCoV extended panel assay file for SYNCT software or other authorized software. ${ }^{29}$

\section{Rheonix COVID-19 MDx Assay}

It is used with the Rheonix Encompass MDx workstation. It is fully automated using reverse transcriptase PCR assays in a single-use cartridge detected using microarray technology. The target gene is amplified in the presence of biotin-tagged primers and the resulting amplicons denatured and flowed over the low-density array of capture probes, which are contained within the CARD cartridge. Following incubation with streptavidin-conjugated horseradish peroxidase and substrate, color-precipitated spots are detected and analyzed via the onboard image capture system. ${ }^{29}$

\section{SARS-CoV-2 ddPCR Test}

It is an RT droplet digital PCR (ddPCR) test. The SARS-CoV-2 nucleic acid is first extracted, isolated, and purified. Using the one-step RT-ddPCR advanced kit for probes, the purified nucleic acid and mastermix RT-ddPCR mixtures are fractionated into up to 20,000 nanoliter-sized droplets in the form of a water-in-oil emulsion in the automated droplet generator or other authorized instrument. The emulsions are then thermocycled to achieve reverse transcription to generate cDNA, followed by target amplification plus probe hydrolysis in each droplet. After PCR, the fluorescence intensity of each droplet is measured in two channels (FAM and HEX) in the droplet reader or other authorized instrument. The fluorescence data are then analyzed by QuantaSoft v1.7 software, QuantaSoft Analysis Pro v1.0 software, or other authorized software to determine the presence of SARS-CoV-2. ${ }^{29}$

\section{Simplexa COVID-19 Direct}

It enables the direct amplification of SARS-CoV-2 RNA from NPS. The product is used with the LIAISON MDX (with LIAISON MDX Studio Software), the direct amplification disc, and fluorescent probes ${ }^{29}$

\section{Test of Cure and Test of Infectivity}

NPS and OPS are not sufficient for either test of cure or test of infectivity ${ }^{36}$ The optimal method for the test of cure most likely will be two consecutive negative real-time RT-PCR tests from rectal swabs; this suggestion is based on the fact that SARS-CoV-1 has been cultured from stool during the 2002-2003 SARS outbreak ${ }^{37-39}$ and SARS-CoV-2 has been cultured from stool during the COVID-19 outbreak ${ }^{40}$ Thus, a rectal swab that is positive by real-time PCR testing suggests that this patient may be shedding viable SARS-CoV-2 in their stools thereby remain infectious. ${ }^{19,37-41} \mathrm{~A}$ study on 20 serial COVID-19 patients indicated that the infectious virus was not isolated from stool samples despite highvirus RNA concentration. ${ }^{42}$ Hence, preferably two consecutive negative real-time RT-PCR tests from both NPS +/-OPS along with rectal swab should be considered as a reliable test of cure and infectivity. However, the utility of any NAAT as a test of cure should be looked with caution, as NAAT cannot differentiate between live or nonviable and dead viral particles.

\section{Problems in Molecular Assays}

Several factors could lead to a negative result in an infected individual, including:

1. Poor quality of the specimen, containing little patient material.

2. Specimens collected late or very early in the infection.

3. The specimen not handled and shipped appropriately.

4. Technical reasons inherent in the test, for example, virus mutation or PCR inhibition. ${ }^{17,21}$

Low-viral loads or high-Ct values can be explained by a variety of mechanisms including inefficient specimen collection, sampling too early or too late in the course of infection, or low-levels of viral shedding overall. ${ }^{21}$

\section{Conclusion}

COVID-19 has emerged as a global catastrophe affecting a large group of population in a small duration of time. Accurate diagnosis of people infected with COVID-19 is essential to curb the 
disease spread. However, the current RT-PCR based diagnostic assays are labor-intensive, time-consuming and costly. So there is a need to develop point-of-care or near point-of-care molecular assays as well as affordable automated diagnostic assay platforms. This development would help in accurate and early diagnosis of infected cases to curb the further spread of this disease to large population and community.

Conflict of Interest

None declared.

\section{References}

1 Loeffelholz MJ, Tang YW. Laboratory diagnosis of emerging human coronavirus infections - the state of the art. Emerg Microbes Infect 2020;9(1):747-756

2 Kaul D. An overview of coronaviruses including the SARS-2 coronavirus - Molecular biology, epidemiology and clinical implications. Curr Med Res Pract 2020;10(2):54-64

3 Ksiazek TG, Erdman D, Goldsmith CS, et al; SARS Working Group. A novel coronavirus associated with severe acute respiratory syndrome. N Engl J Med 2003;348(20):1953-1966

4 Assiri A, Al-Tawfiq JA, Al-Rabeeah AA, et al. Epidemiological, demographic, and clinical characteristics of 47 cases of Middle East respiratory syndrome coronavirus disease from Saudi Arabia: a descriptive study. Lancet Infect Dis 2013;13(9):752-761

5 COVID-19 situation reports. Who.int. Available at: https:// www.who.int/emergencies/diseases/novel-coronavirus-2019/ situation- reports/. Accessed May 21, 2020

6 Vashist SK. In vitro diagnostic assays for COVID-19: recent advances and emerging trends. Diagnostics (Basel) 2020;10(4):202

7 Singhal T. A review of Coronavirus disease-2019 (COVID-19) Indian J Pediatr 2020;87(4):281-286

8 Sahin A. 2019 novel Coronavirus (COVID-19) outbreak: a review of the current literature. Eurasian J Med Oncol 2020. Doi: 10.14744/ejmo.2020.12220

9 Huang C, Wang Y, Li X, et al. Clinical features of patients infected with 2019 novel coronavirus in Wuhan, China. Lancet 2020;395(10223):497-506

10 Indian Council of Medical Research (ICMR). Main.icmr.nic. in. Available at: https://main.icmr.nic.in/sites/default/files/ upload_documents/Testing_Strategy_v5_18052020.pdf. Accessed May 21, 2020

11 Hanson K, Caliendo A, Arias C, et al. Infectious Diseases Society of America Guidelines on the Diagnosis of COVID-19. Idsociety. org. Available at: https://www.idsociety.org/globalassets/idsa/ practice-guidelines/covid-19/diagnostics/idsa-covid-19guideline_dx_version-1.0.1.pdf. Accessed May 21, 2020

12 Indian Council of Medical Research (ICMR). Icmr.gov.in. Available at: https://www.icmr.gov.in/pdf/covid/labs/COVID_ Testing_Labs_19052020.pdf. Accessed May 21, 2020

13 Central Pollution Control Board. Guidelines for Handling, Treatment and Disposal of Waste Generated during Treatment/ Diagnosis/Quarantine of COVID-19 patients. Available at: https:// ncdc.gov.in/showfile.php?lid=551. Accessed May 21, 2020

14 Ministry of Health and Family Welfare. Guidelines for BMW Management at Quarantine Facilities for COVID-19. Available at: https://www.mohfw.gov.in/pdf/90542653311584546120quartineguidelines.pdf. Accessed May 21, 2020
15 World Health Organization. Infection Prevention and Control during Health Care when COVID-19 is Suspected: Interim Guidance, 19 March 2020. Available at: https://apps.who.int/ iris/bitstream/handle/10665/331495/WHO-2019-nCoV-IPC2020.3-eng.pdf. Accessed May 21, 2020

16 Tang YW, Schmitz JE, Persing DH, Stratton CW. Laboratory diagnosis of COVID-19: current issues and challenges. J Clin Microbiol 2020;58(6):e00512-20

17 World Health Organization. Laboratory Testing Strategy Recommendations for COVID-19: Interim Guidance, 22 March 2020. Available at: https://apps.who.int/iris/bitstream/handle/10665/331509/WHO-COVID-19-lab_testing-2020.1-eng. pdf. Accessed May 21, 2020

18 Centre for Disease Control and Prevention. Interim Guidelines for Collecting, Handling, and Testing Clinical Specimens from Persons for Coronavirus Disease (COVID-19). Available at: https://www.cdc.gov/coronavirus/2019- ncov/lab/guidelinesclinical-specimens.html. Accessed May 21, 2020

19 Wang W, Xu Y, Gao R, et al. Detection of SARS-CoV-2 in Different Types of Clinical Specimens. JAMA 2020. Doi: 10.1001/jama. 2020.3786

20 World Health Organization. Molecular Assays to Diagnose COVID-19: Summary Table of Available Protocols. Available at: https://www.who.int/who-documents- detail/molecular-assays-to-diagnose-covid-19-summary-table-of-available-protocolsPublished 2020. Accessed May 21, 2020

21 Loeffelholz MJ, Alland D, Butler-Wu SM, et al. Multicenter evaluation of the Cepheid Xpert Xpress SARS-CoV-2 test. J Clin Microbiol 2020. Doi: $10.1128 / \mathrm{jcm} .00926-20$

22 World Health Organization. Laboratory Biosafety Guidance Related to Coronavirus Disease 2019 (COVID-19): Interim Guidance, 12 February 2020. Available at: https://apps. who.int/iris/bitstream/handle/10665/332076/WHO-WPEGIH-2020.3- eng.pdf. Accessed May 21, 2020

23 Udugama B, Kadhiresan P, Kozlowski HN, et al. Diagnosing COVID-19: The Disease and Tools for Detection. ACS Nano 2020;14(4):3822-3835

24 Shen M, Zhou Y, Ye J, et al. Recent advances and perspectives of nucleic acid detection for coronavirus. J Pharm Anal 2020;10(2):97-101

25 Lamb L, Bartolone S, Ward E, Chancellor M, Rapid detection of novel Coronavirus (COVID19) by reverse transcription-loop-mediated isothermal amplification. SSRN Electronic Journal 2020. Doi: 10.2139/ssrn.3539654

26 Yu L, Wu S, Hao X, et al. Rapid detection of COVID-19 coronavirus using a reverse transcriptional loop-mediated isothermal amplification (RT-LAMP) diagnostic platform. Clin Chem 2020. Doi: 10.1093/clinchem/hvaa102

27 Zhang Y, Odiwuor N, Xiong J, et al. Rapid molecular detection of SARS-CoV-2 (COVID-19) virus RNA using colorimetric LAMP. medRxiv 2020. Doi:10(2020.02):26-0028373

28 Yang W, Dang X, Wang Q, et al. Rapid detection of SARS-CoV-2 using reverse transcription RT-LAMP method. medRxiv2020;Doi: $10.1101 / 2020.03 .02 .20030130$

29 United States Food and Drug Administration. Available at: https://www.fda.gov/medical-devices/emergency-situations-medical- devices/emergency-useauthorizations\#coronavirus. Accessed May 21, 2020

30 World Health Organization. Available at: https://www.who. int/diagnostics_laboratory/EUL/en/. Accessed May 21, 2020

31 Indian Council of Medical Research. Available at: https://www. icmr.gov.in/pdf/covid/kits/Real_time_PCR_tests_01052020. pdf. Accessed May 21, 2020 
32 Foundation for Innovative New Diagnostics. Available at: https://www.finddx.org/covid-19/pipeline/?section=molecular-assays\#diag_tab. Accessed May 21, 2020

33 Wang X, Zhong M, Liu Y, et al. Rapid and sensitive detection of COVID-19 using CRISPR/Cas12a-based detection with naked eye readout, CRISPR/Cas12a-NER. Sci Bull (Beijing) 2020. Doi: 10.1016/j.scib.2020.04.041

34 Hou T, Zeng W, Yang M, et al. Development and evaluation of A CRISPR-based diagnostic for 2019-novel Coronavirus. medRxiv 2020. Doi: 10.1101/2020.02.22.20025460

35 Kellner MJ, Koob JG, Gootenberg JS, Abudayyeh OO, Zhang F. SHERLOCK: nucleic acid detection with CRISPR nucleases. Nat Protoc 2019;14(10):2986-3012

36 Lan L, Xu D, Ye G, et al. Positive RT-PCR Test results in patients recovered From COVID-19. JAMA 2020;323(15):1502

37 Cheng PK, Wong DA, Tong LK, et al. Viral shedding patterns of coronavirus in patients with probable severe acute respiratory syndrome. Lancet 2004;363(9422):1699-1700
38 Leung WK, To KF, Chan PK, et al. Enteric involvement of severe acute respiratory syndrome-associated coronavirus infection. Gastroenterology 2003;125(4):1011-1017

39 Xu D, Zhang Z, Jin L, et al. Persistent shedding of viable SARS$\mathrm{CoV}$ in urine and stool of SARS patients during the convalescent phase. Eur J Clin Microbiol Infect Dis 2005;24(3):165-171

40 Zhang W, Du RH, Li B, et al. Molecular and serological investigation of 2019-nCoV infected patients: implication of multiple shedding routes. Emerg Microbes Infect 2020;9(1):386-389

41 Yeo C, Kaushal S, Yeo D. Enteric involvement of coronaviruses: is faecal-oral transmission of SARS-CoV-2 possible? Lancet Gastroenterol Hepatol 2020;5(4):335-337

42 Wölfel R, Corman VM, Guggemos W, et al. Virological assessment of hospitalized patients with COVID-2019. Nature 2020;581(7809):465-469 